\title{
A Study on Quark-Gluon Plasma Equation of State Using Generalized Uncertainty Principle
}

\author{
Nabil Mohamed El Naggar, Lotfy Ibrahim Abou-Salem, \\ Ibrahim Abdelbasit Elmashad, Ahmed Farag Ali \\ Physics Department, Faculty of Science, Benha University, Benha, Egypt \\ Email: loutfy.Abousalem@fsc.bu.edu.eg, ibrahim.elmashad@fsc.bu.edu.eg,ahmed.ali@fsc.bu.edu.eg
}

Received February 6, 2013; revised March 8, 2013; accepted March 20, 2013

Copyright (C) 2013 Nabil Mohamed El Naggar et al. This is an open access article distributed under the Creative Commons Attribution License, which permits unrestricted use, distribution, and reproduction in any medium, provided the original work is properly cited.

\begin{abstract}
The effects of Generalized Uncertainty Principle, which has been predicted by various theories of quantum gravity replacing the Heisenberg's uncertainty principle near the Planck scale, on the thermodynamics of ideal Quark-Gluon Plasma (QGP) consisting of two and three flavors are included. There is a clear effect on thermodynamical quantities like the pressure and the energy density which means that a different effect from quantum gravity may be used in enhancement the theoretical results for Quark-Gluon Plasma state of matter. This effect looks like the technique used in lattice QCD simulation. We determine the value of the bag parameter from fitting lattice QCD data and a physical interpretation to the negative bag pressure is introduced.
\end{abstract}

Keywords: Generalized Uncertainty Principle

\section{Introduction}

Since various theories of quantum gravity predict essential modifications in the Heisenberg's uncertainty principle near the Planck scale, We utilize the proposed generalized uncertainty principle (GUP), which proved compatible with string theory, doubly special relativity and black hole physics.

Recently, a new model of GUP was proposed [1-3]. It predicts a maximum observable momentum and a minimal measurable length. Accordingly, $\left[x_{i}, x_{j}\right]=\left[p_{i}, p_{j}\right]=0$ (via the Jacobi identity) results in.

$$
\begin{aligned}
& {\left[x_{i}, p_{j}\right]} \\
& =i \hbar\left[\delta_{i j}-\alpha\left(p \delta_{i j}+\frac{p_{i} p_{j}}{p}\right)+\alpha^{2}\left(p^{2} \delta_{i j}+3 p_{i} p_{j}\right)\right],
\end{aligned}
$$

where $\alpha=\alpha_{0} / M_{p} c=\alpha_{0} \ell_{p} / \hbar$ and $M_{p} c^{2}$ stand for Planck energy. $M_{p}$ and $\ell_{p}$ is Planck mass and length, respectively. $\alpha_{0}$ sets on the upper and lower bounds to $\alpha$. Apparently, Equation (1) imply the existence of a minimum measurable length and a maximum measurable momentum

$$
\begin{aligned}
& \Delta x_{\min } \approx \alpha_{0} \ell_{p}, \\
& \Delta p_{\max } \approx \frac{M_{p} C}{\alpha_{0}}
\end{aligned}
$$

where $\Delta x \geq \Delta x_{\min }$ and $\Delta p \leq \Delta p_{\max }$. Accordingly, for a particle having a distant origin and an energy scale comparable to the Planck's one, the momentum would be a subject of a modification [1-3].

$$
p_{i}=p_{0 i}\left(1-\alpha p_{0}+2 \alpha^{2} p_{0}^{2}\right),
$$

where $x_{i}=x_{0 i}$ and $p_{0 j}$ satisfy the canonical commutation relations $\left[x_{0 i}, p_{0 j}\right]=i \hbar \delta_{i j}$ and simultaneously fulfil Equation (1). Here, $p_{0 i}$ can be interpreted as the momentum at low energies (having the standard representation in position space, i.e. $\left.p_{0 i}=-i \hbar \partial / \partial x_{0 i}\right)$ and $p_{i}$ as that at high energies.

The proposed GUP is assuming that the space is discrete, and that all measurable lengths are quantized in units of a fundamental minimum and measurable length. The latter can be as short as the Planck length $[1,2]$. In order to support the idea of this procedure, we can mention that similar quantization of the length (spatial dimensions) has been studied in context of loop quantum gravity [4]. Furthermore, it has been suggested recently [5] that the GUP implications can be measured directly in quantum optics lab which seems to confirm the theoretical predictions [6-8].

Since the GUP apparently modifies the fundamental commutator bracket between position and momentum operators, then it is natural to expect that this would result in considerable modifications in the Hamiltonian. 
Furthermore, it would affect a host of quantum phenomena, as well. It is important to make a quantitative study of these effects. In a series of earlier papers, the effects of GUP was investigated on atomic and condensed matter systems [2,6-8], on the weak equivalence principle (WEP), and on the Liouville theorem (LT) in statistical mechanics [9]. For instance, it has been found that GUP can potentially explain the small observed violations of the WEP in neutron interferometry experiments [10-12]. Also, it can predict the existence of a modified invariant phase space which is relevant to the Liouville theorem.It seems that this approach accordingly modifies almost all mechanical Hamiltonians. Therefore, it can be implemented on studying the thermodynamics. In this paper, we present a study for the impact of the GUP on Quark Gluon Plasma (QGP). We calculate the corrections to various thermodynamic quantities, like energy density, pressure, equation of state and entropy.

This paper is organized as follows. In Section 2, we review QGP, briefly. In Section 3, we investigate the thermodynamics of QGP and estimate the impact of GUP approach. We give our conclusions in Section 4.

\section{Thermodynamics of Quark-Gluon Plasma}

In this section, we briefly review the thermodynamics of QGP consists of fermions. At finite temperature $T$ and chemical potential $\mu$, the grand-canonical partition function $Z_{F}$ for non-interacting massive fermions with $g$ internal degrees of freedom is given as [13]

$$
\begin{aligned}
& Z_{F}=\prod_{k}\left[\sum_{l=0,1} \exp \left(\frac{-l(E(k)-\mu)}{T}\right)\right]^{g} \\
& Z_{F}=\prod_{k}\left[1+\exp \frac{-(E(k)-\mu)}{T}\right]^{g}
\end{aligned}
$$

where $k$ is the momentum of the particle and $l$ is the occupation number for each quantum state with energy $E(k)=\sqrt{k^{2}+m^{2}}$. Here the infinite product is taken for all possible momentum states.

Then the grand potential of a non-interacting massive fermion gas in a vanishing chemical potential is

$$
\begin{aligned}
& \frac{\Omega(T, V, 0)}{V}=g \int_{0}^{\infty} \frac{\mathrm{d}^{3} k}{(2 \pi)^{3}} T \ln \left[1+\mathrm{e}^{\frac{-E(k)}{T}}\right] \cdot \begin{array}{c}
\sum_{k} \rightarrow \frac{V}{(2 \pi)^{3}} \int_{0}^{\infty} \mathrm{d}^{3} k \rightarrow \frac{V}{2 \pi^{2}} \int_{0}^{\infty} \frac{k^{2} \mathrm{~d} k}{(1-\alpha k)^{4}} \cdot \\
\text { Therefore, the partition function reads }
\end{array} \\
& \ln z_{F}=-\frac{V g}{2 \pi^{2}} \int_{0}^{\infty} k^{2} \frac{\ln \left[1+\exp \left(-\frac{E(k)}{T}\right)\right]}{(1-\alpha k)^{4}} \mathrm{~d} k=-\frac{V g}{2 \pi^{2}} \int_{0}^{\infty} k^{2} \frac{\ln \left[1+\exp \left(-\frac{k}{T}(1-2 \alpha k)^{1 / 2}\right)\right]}{(1-\alpha k)^{4}} \mathrm{~d} k, \\
& =-\frac{V g}{2 \pi^{2}}\left[\left.\frac{k^{3}}{3(1-\alpha k)^{3}} \ln \left[1+\exp \left(-\frac{k}{T}(1-2 \alpha k)^{1 / 2}\right)\right]\right|_{0} ^{\infty}+\frac{V g}{2 \pi^{2}} \int_{0}^{\infty} \frac{k^{3}}{3(1-\alpha k)^{3}} \frac{\frac{1}{T} \exp \left(-\frac{k}{T}(1-2 \alpha k)^{1 / 2}\right)}{1+\exp \left(-\frac{k}{T}(1-2 \alpha k)^{1 / 2}\right)} \cdot \frac{1-3 \alpha k}{\left.(1-2 \alpha k)^{1 / 2}\right] \mathrm{d} k .}\right.
\end{aligned}
$$

The value of this integral reads [13]

$$
\begin{aligned}
& \frac{\Omega(T, V, 0)}{V} \\
& =-\frac{7}{8} g\left[\frac{\pi^{2}}{90} T^{4}-\frac{M^{2} T^{2}}{42}-\frac{M^{4}}{56 \pi^{2}}\left(\ln \left(\frac{M^{2}}{(\pi T)^{2}}+C\right)\right)+\cdots\right],
\end{aligned}
$$

with $C=2 \gamma-3 / 2 \simeq-0.346$, and $\gamma$, the Euler constant, defined as [13]

$$
\gamma=\lim _{n \rightarrow \infty}\left(\sum_{k=1}^{n} \frac{1}{k}-\ln n\right)=\sum_{n=2}^{\infty}(-1)^{n} \frac{\zeta(n)}{n}=0.5772156649 \ldots
$$

For simplicity we consider a non-interacting massless fermion gas in a vanishing chemical potential. Then the grand potential reads

$$
\frac{\Omega}{V}=g \int_{0}^{\infty} \frac{\mathrm{d}^{3} k}{(2 \pi)^{3}} T \ln \left[1+\exp \frac{-E(k)}{T}\right]=-g \frac{7}{8} \frac{\pi^{2}}{90} T^{4} .
$$

Therefore, pressure $P$ and energy density, $\varepsilon$, of hadronic state can be deduced

$$
P_{H}=g_{H} \frac{7}{8} \frac{\pi^{2}}{90} T^{4}, \varepsilon_{H}=3 g_{q} \frac{7}{8} \frac{\pi^{2}}{90} T^{4} \text {. }
$$

\section{Thermodynamics of Quark-Gluon Plasma with Effect of GUP}

For a particle of mass $M$ having a distant origin and an energy scale comparable to the Planck's one, the momentum would be a subject of a tiny modification and so the dispersion relation would too. According to GUPapproach, the dispersion relation in the co moving frame reads

$$
E^{2}(k)=k^{2} c^{2}(1-2 \alpha k)+M^{2} c^{4} .
$$

For simplicity we use natural units in which $\hbar=c=1$ and consider a massless pion gas

$$
E(k)=k(1-2 \alpha k)^{1 / 2} .
$$

For large volume, the sum over all states of single particle can be rewritten in terms of an integral [14] 
It is obvious that the first term in Equation (11) vanishes. Thus

$$
\ln z_{F}=\frac{V g}{2 \pi^{2}} \int_{0}^{\infty} \frac{k^{3}}{3(1-\alpha k)^{3}} \frac{\frac{1}{T}\left[\frac{1-3 \alpha k}{(1-2 \alpha k)^{1 / 2}}\right]}{\exp \left(-\frac{k}{T}(1-2 \alpha k)^{1 / 2}\right)+1} \mathrm{~d} k .
$$

Let $x=\frac{k}{T}(1-2 \alpha k)^{1 / 2}$ so that $\mathrm{d} x=\frac{1}{T} \frac{1-3 \alpha k}{(1-2 \alpha k)^{1 / 2}} \mathrm{~d} k$ and the integral becomes

$$
\ln z_{F}=\frac{V g}{2 \pi^{2}} \int_{0}^{\infty} \frac{k^{3}}{3(1-\alpha k)^{3}} \frac{\mathrm{d} x}{\mathrm{e}^{(x)}+1} .
$$

Apparently, as we are interested in the terms containing the first order of $\alpha$, so $x$ can be approximated as follows

$$
x=\frac{k}{T}(1-2 \alpha k)^{1 / 2} \approx \frac{k(1-\alpha k)}{T} .
$$

Then

$$
k=x T+\alpha k^{2} \approx x T+\alpha\left(x^{2} T^{2}+2 \alpha x T k^{2}+\alpha^{2} k^{4}\right) .
$$

when ignoring higher orders of $\alpha$,

$$
k \approx x T(1+\alpha x T) .
$$

Then the partition function becomes

$$
\ln z_{F}=\frac{V g}{2 \pi^{2}} \int_{0}^{\infty} \frac{1}{3} x^{3} T^{3} \frac{(1+\alpha x T)^{3}}{(1-\alpha x T)^{3}} \frac{\mathrm{d} x}{\mathrm{e}^{x}+1} .
$$

It is apparent that the integral contains Maclaurin series, which are

$$
\begin{aligned}
\frac{(1+\alpha x T)^{3}}{(1-\alpha x T)^{3}} & =1+6 \alpha x T+[\cdots] \alpha^{2} x^{2} T^{2} \\
& +[\cdots] \alpha^{3} x^{3} T^{3}+\cdots
\end{aligned}
$$

when ignoring terms containing $\alpha$ with order $\geq 2$, then

$$
\ln z_{F}=\frac{V g}{6 \pi^{2}}\left[\int_{0}^{\infty} T^{3} \frac{x^{3} \mathrm{~d} x}{\mathrm{e}^{x}+1}+\int_{0}^{\infty} 6 \alpha T^{4} \frac{x^{4} \mathrm{~d} x}{\mathrm{e}^{x}-1}\right] .
$$

The partition function is related to the grand canonical potential, $\ln z_{F}=-\Omega / T$, so we have

$$
\begin{aligned}
& \frac{\Omega}{V}=-\frac{g}{6 \pi^{2}} T\left[T^{3} \int_{0}^{\infty} \frac{x^{3} \mathrm{~d} x}{\mathrm{e}^{x}-1}+6 \alpha T^{4} \int_{0}^{\infty} \frac{x^{4} \mathrm{~d} x}{\mathrm{e}^{x}-1}\right] \\
& =\frac{-T g}{6 \pi^{2}}\left[\int_{0}^{\infty} \frac{x^{3} T^{3} \mathrm{~d} x}{\left(\mathrm{e}^{x}-1\right)}\right]+\frac{\alpha g T^{5}}{\pi^{2}}\left[\int_{0}^{\infty} \frac{x^{4} \mathrm{~d} x}{\left(\mathrm{e}^{x}-1\right)}\right], \\
& =-\frac{g}{6 \pi^{2}} T^{4}\left[\Gamma(5) I(0)_{5}^{+}\right]-\alpha \frac{g}{\pi^{2}} T^{5}\left[\Gamma(6) I(0)_{6}^{+}\right]
\end{aligned}
$$

where $I(0)_{n}^{ \pm}$are Bose and Fermi integrals. Substituting by the value of these integrals in (20) we have

$$
\begin{aligned}
& \frac{\Omega}{V}=-g \frac{\pi^{2}}{90} \frac{7}{8} T^{4}-\frac{45}{2} \alpha \frac{g}{\pi^{2}} T^{5} \zeta(5) \\
& \frac{\Omega}{V}=-g \frac{\pi^{2}}{90} \frac{7}{8} T^{4}-g \alpha_{2} T^{5}
\end{aligned}
$$

where $\alpha_{2}=\frac{45 \alpha \zeta(5)}{2 \pi^{2}}$. But the pressure is directly related to the grand canonical potential, $P=-\Omega / V$, so we obtain

$$
P_{H}=g_{q} \frac{7}{8} \frac{\pi^{2}}{90} T^{4}+g_{q} \alpha_{2} T^{5} .
$$

The energy density is related with the pressure by the relation $(\varepsilon=3 P)$ and the entropy density is given by the derivative of pressure with respect to temperature $T$

$$
\varepsilon_{H}=3 g_{q} \frac{7}{8} \frac{\pi^{2}}{90} T^{4}+3 g_{q} \alpha_{2} T^{5} .
$$

Taking into consideration the relevant degrees of freedom, it is obvious that this set of equations, Equations (23) and (24), is valid in hadronic state.

Similarly, the grand-canonical partition function $z_{B}$ for non-interacting massive bosons with $g$ internal degrees of freedom is given as

$$
\begin{aligned}
\ln z_{B} & =\prod_{k}\left[\sum_{l=0}^{\infty} \exp \left(-l \frac{E(k)-\mu}{T}\right)\right]^{g} \\
\ln z_{B} & =\prod_{k}\left[1-\exp \frac{(E(k)-\mu)}{T}\right]^{-g} .
\end{aligned}
$$

Then the grand canonical potential reads

$$
\begin{aligned}
\frac{\Omega}{V} & =-g \frac{\pi^{2}}{90} T^{4}-24 \alpha \frac{g}{\pi^{2}} T^{5} \zeta(5) \\
& =-g \frac{\pi^{2}}{90} T^{4}-g \alpha_{1} T^{5}
\end{aligned}
$$

where $\alpha_{1}=24 \alpha \zeta(5) / \pi^{2}$. The pressure is directly related to the grand canonical potential, $P=-\Omega / V$. Then, in the hadronic phase

$$
P_{H}=g \frac{\pi^{2}}{90} T^{4}+g \alpha_{1} T^{5} .
$$

In a massless ideal hadronic gas, the energy density is related with the pressure by the equation of state, $\varepsilon_{H}=3 P_{H}$, and the entropy density is given by the derivative of pressure with respect to temperature $T$

$$
\varepsilon_{H}=3 g \frac{\pi^{2}}{90} T^{4}+3 g \alpha_{1} T^{5} .
$$


Now we will derive the QGP equation of state consists of free massless quarks and gluons. The total grand canonical partition function of the hadronic matter can be obtained by combining the grand partition functions coming from the contribution of fermions (quarks), bosons (gluons) and vacuum. It reads [15]

$$
\ln Z_{Q G P}=\ln Z_{F}+\ln Z_{B}+\ln Z_{v}
$$

where $\ln Z_{F}, \ln Z_{B}$ and $\ln Z_{v}$ are the grand canonical partition functions of quarks, gluons and vacuum respectively. Since the value of vacuum partition function equals $\ln Z_{v}=-\frac{V B}{T}$, so Equation (30) becomes

$$
\ln Z_{Q G P}=\ln Z_{F}+\ln Z_{B}-\frac{V B}{T} .
$$

From Equation (30) and equation $\ln Z=\frac{-\Omega}{T}$ we have

$$
\left.\frac{\Omega}{V}\right|_{Q G P}=\left.\frac{\Omega}{V}\right|_{\text {gluons }}+\left.\frac{\Omega}{V}\right|_{\text {quarks }}+\left.\frac{\Omega}{V}\right|_{\text {vacuum }} .
$$

Substituting from Equations (21) and (28) into Equation (32) we have

$$
\begin{aligned}
\frac{\Omega}{V} & =-g_{g} \frac{\pi^{2}}{90} T^{4}-g_{g} \alpha_{1} T^{5}-g_{q} \frac{7}{8} \frac{\pi^{2}}{90} T^{4}-g_{q} \alpha_{2} T^{5}+B \\
& =-\left(g_{g}+\frac{7}{8} g_{q}\right) \frac{\pi^{2}}{90} T^{4}-\left(g_{g} \alpha_{1}+g_{q} \alpha_{2}\right) T^{5}+B .
\end{aligned}
$$

Thus, the QGP pressure $P=-\frac{\Omega}{V}$ reads

$$
P_{Q G P}=g_{Q G P} \frac{\pi^{2}}{90} T^{4}+\left(g_{g} \alpha_{1}+g_{q} \alpha_{2}\right) T^{5}-B
$$

where $g_{Q G P}=g_{g}+\frac{7}{8} g_{q}$ are the QGP degenercy. Then the energy density reads

$$
\varepsilon_{Q G P}=3 g_{Q G P} \frac{\pi^{2}}{90} T^{4}+3\left(g_{g} \alpha_{1}+g_{q} \alpha_{2}\right) T^{5}+B .
$$

\section{Results and Conclusions}

\subsection{Comparison to Lattice QCD Simulations with Massless Quarks}

Generally, the value of pressure and the energy density depends on the temperature, the degenercy and the bag pressure. In this work, another factor added to them which originating from quantum gravity effect. The second term in Equation (23) which includes $\alpha_{2}$ gives a positive contribution to the pressure. As discussed in [12], the exact bound state on $\alpha$ can be obtained by comparing with observations and experiments [16]. The gamma rays burst would allow us to set an upper value for the GUP-charactering parameter $\alpha$. We will take the value of $\alpha \sim 0.005 \mathrm{GeV}^{-1}$ which equals to the half value that corresponding to the upper bound for $\alpha_{0}$ [6].

This term give a good results with lattice QCD due to the rapid increase contribution to the pressure. The bag parameter, $B$, has more than one method to be determined. One of them based on fitting the pressure or the energy density with lattice QCD. The problem appears when one start from fitting the pressure function with Equation (33), one obtains a good qualitative agreement with lattice QCD results for admitting positive values of the bag constant. The positive bag constant needed in Equation (33) to fit the pressure leads, however, to an incorrect behavior of energy density. The same situation occurs when we start to calculate the value of, $B$, from fitting the energy density with Equation (34), this gives us a negative value of bag constant. Thus the value of, $B$, depending on whether we start from fitting the pressure or the energy density [17]. To overcome this problem, a modification of bag model was introduced to solve this problem [18]. In this technique, the fundamental thermodynamical relation between the pressure and the energy density is used which reads [14]

$$
\varepsilon(T)=T \frac{\mathrm{d} P}{\mathrm{~d} T}-P(T) .
$$

Since Equation (35) is a 1st order partial differential equation, so if the energy density function known, then the general solution of Equation (35) gives us an arbitrary integration constant which surely depends on temperature. Then the new pressure function can be fitted from lattice QCD results. In Refs. [17,18], this term had a linear dependence on temperature and the bag model carries the name (A-Bag model). This method was discussed at first time in Ref. [19]. For the energy density in the form of Equation (34), i.e. GUP is included, the general solution of Equation (35) reads

$$
P_{Q G P}=g_{Q G P} \frac{\pi^{2}}{90} T^{4}+\frac{3}{4}\left(g_{g} \alpha_{1}+g_{q} \alpha_{2}\right) T^{5}-B+A T .
$$

It is clear that beside the linear term, there is also another term, which can be easily obtained from comparing Equations (33) and (36), equals $-\left(\left(g_{g} \alpha_{1}+g_{q} \alpha_{2}\right) T^{5} / 4\right)$. This term due to quantum gravity effect and gives a negative contribution to the QGP pressure.

To get a good fitting, we will consider the suppression factor of the Stefan Boltzmann constant $\sigma_{S B}=3 g_{Q G P} \frac{\pi^{2}}{90}$. The quasi-particle approach [20] is used. In this approach, the system of interacting gluons is treated as a gas of non-interacting quasiparticles with gluon quantum numbers, but with thermal mass (i.e. $m(T)$ ). The modified SB constant $\sigma$ equals to [17] 


$$
\sigma=\kappa(a) \sigma_{S B}
$$

where $a$ has a direct connection with temperature and the thermal mass. The expression (37) was used for all $T \geq T_{c}$ to keep the high temperature behavior of both pressure and energy density in agreement with lattice QCD. In Figure 1, the fitting of QGP pressure and energy density from Lattice QCD results [21] Using Equations (34) and (36). We take $n_{f}=2, g_{O G P}=37$, $g_{q}=24, g_{g}=16, g_{\pi}=8,[13], T_{c}=0.150 \mathrm{GeV}$ and $\alpha=0.005 \mathrm{GeV}^{-1}$. The fitting of energy density gives us the value of bag constant $B \simeq-4.4 T_{c}^{4}$ as shown in Figure 1.

Since we take the chiral limit $\left(m_{q}=0\right)$, then the dominant excitations in the hadronic phase is the massless pions, while that in the QGP is the massless quark and gluon. At extremely high temperature, the typical momenta of quarks and gluons are high and the running coupling $\left(\alpha_{\mathrm{s}}\right)$ becomes so weak due to asymptotic freedom. Thus we can assume a free pion gas (free Quark-Gluon) in the low (high) temperature limit as a first approximation [13]. From the phase equilibrium condition $P_{H}\left(T_{c}\right)=P_{Q G P}\left(T_{c}\right)$, the critical point can be obtained by equating Equations (28) and (36) at $T_{c}$. Substituting the value of $B$, we obtain the value of $A$ which equals $A \simeq-6.76 T_{c}^{3}$. Then Substituting the value of $A$ and $B$, we get the behavior of pressure showed in Figure 2 which is in a quit good qualitative behavior comparing with lattice QCD.
In Figure 3, the fitting of QGP pressure and energy density from Lattice QCD results [21] Using Equations (34) and (36). We take $n_{f}=3, g_{Q G P}=47.5, g_{q}=36$, $g_{g}=16, g_{\pi}=8 \quad[13], T_{c}=0.150 \mathrm{GeV}$ and $\alpha=0.005 \mathrm{GeV}^{-1}$. The fitting of energy density gives us the value of bag constant $B \simeq-5.15 T_{c}^{4}$ as shown in Figure 3.

Substituting the value of $B$, we obtain the value of $A$ which equals $A \simeq-8.42 T_{c}^{3}$. Then Substituting the value of $A$ and $B$, we get the behavior of pressure showed in Figure 4 which is in a good qualitative behavior comparing with lattice QCD. The value of suppressed factor in both cases (i.e. $n_{f}=2$ and $n_{f}=3$ ) eqals

$$
\kappa(a) \simeq 0.78 \text {. }
$$

\subsection{Discussion on the Negative Bag Pressure}

Since the bag pressure (vacuum pressure) is related mainly to the confinement phenomenon of hadrons, we need to discuss the picture of confinement. It is believed that all strongly interacting particles are made of quarks and gluons which are color charged. However, all observable physical states formed from them are color neutral. This means that the true vacuum abhors color [15]. Any vacuum in which colored particles can exist as individual entities and so move freely is called "perturbative vacuum". The difference between the perturbative vacuum and the true vacuum, in which we live, is the amount of energy density in the regions of space [15]. In

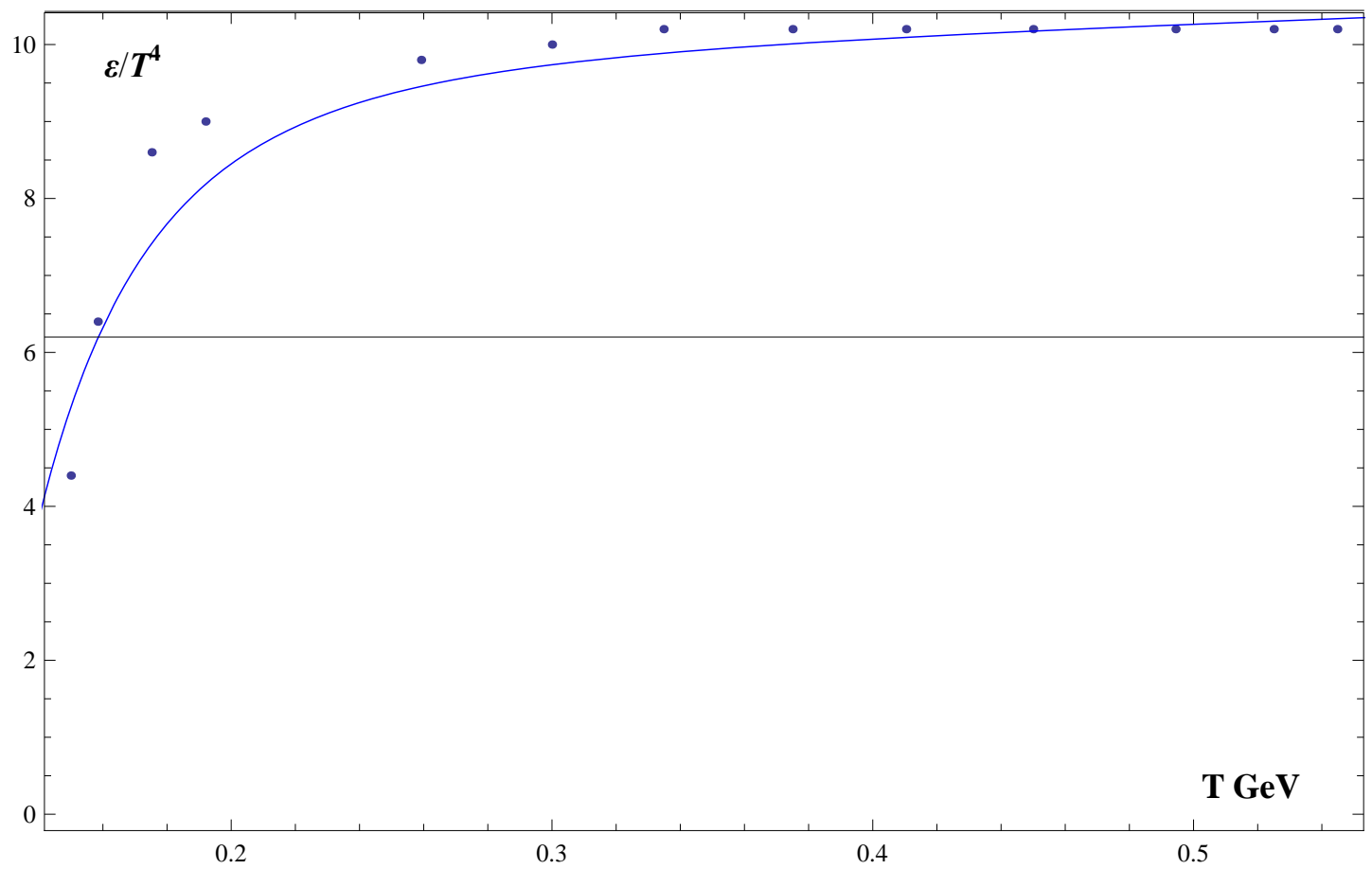

Figure 1. The solid curve gives the fitting of energy density normalized to $T^{4}$ from lattice QCD results for two flavors $n_{f}=2$. 


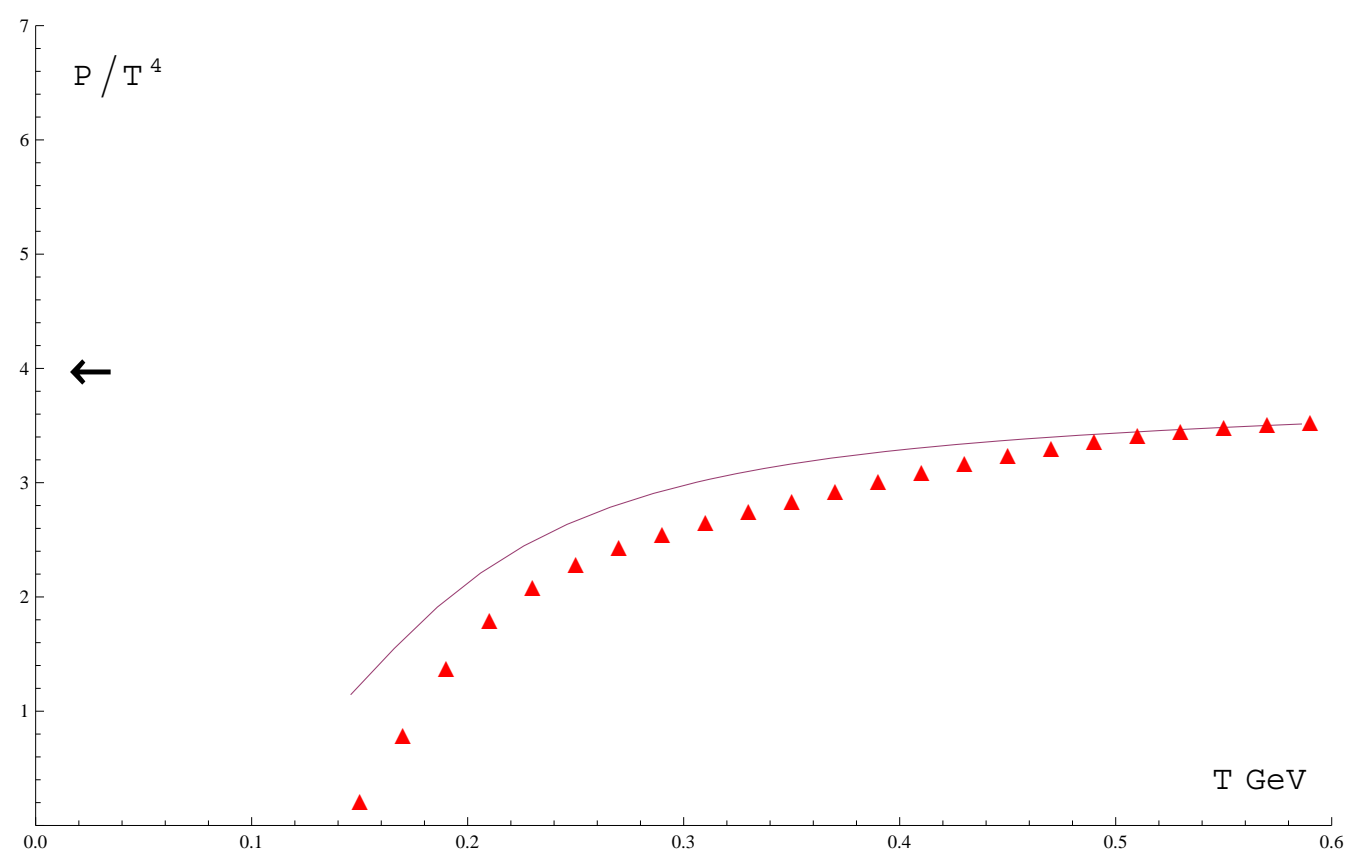

Figure 2. The solid curve gives the pressure normalized to $T^{4}$ comparing with lattice QCD results for two flavors $n_{f}=2$.



Figure 3. The solid curve gives the fitting of energy density normalized to $T^{4}$ from lattice QCD results for three flavors $\boldsymbol{n}_{f}=\mathbf{3}$.

the true vacuum, color-charged quarks and gluons are confined but under extreme conditions of temperature and density, a transition to a deconfinement state of matter is possible. This picture of hadronic interactions is consistent and justifies the perturbative approach to QCD interactions. This allows us to describe hadrons as "bags". So, we need to melt the confining structure to able to move color charges within a region of space. For a first-order phase transition, the two phases have a difference in energy density, the latent heat per unit volume, $B$, equivalent to $\left(\varepsilon_{Q G P}-\varepsilon_{H G}\right)$ [15]. According to the calculated frame work, which was proposed by Bololiubov, independent quarks confined by a static Lorentz-scalar potential with infinite walls was considered. Thus, it was 


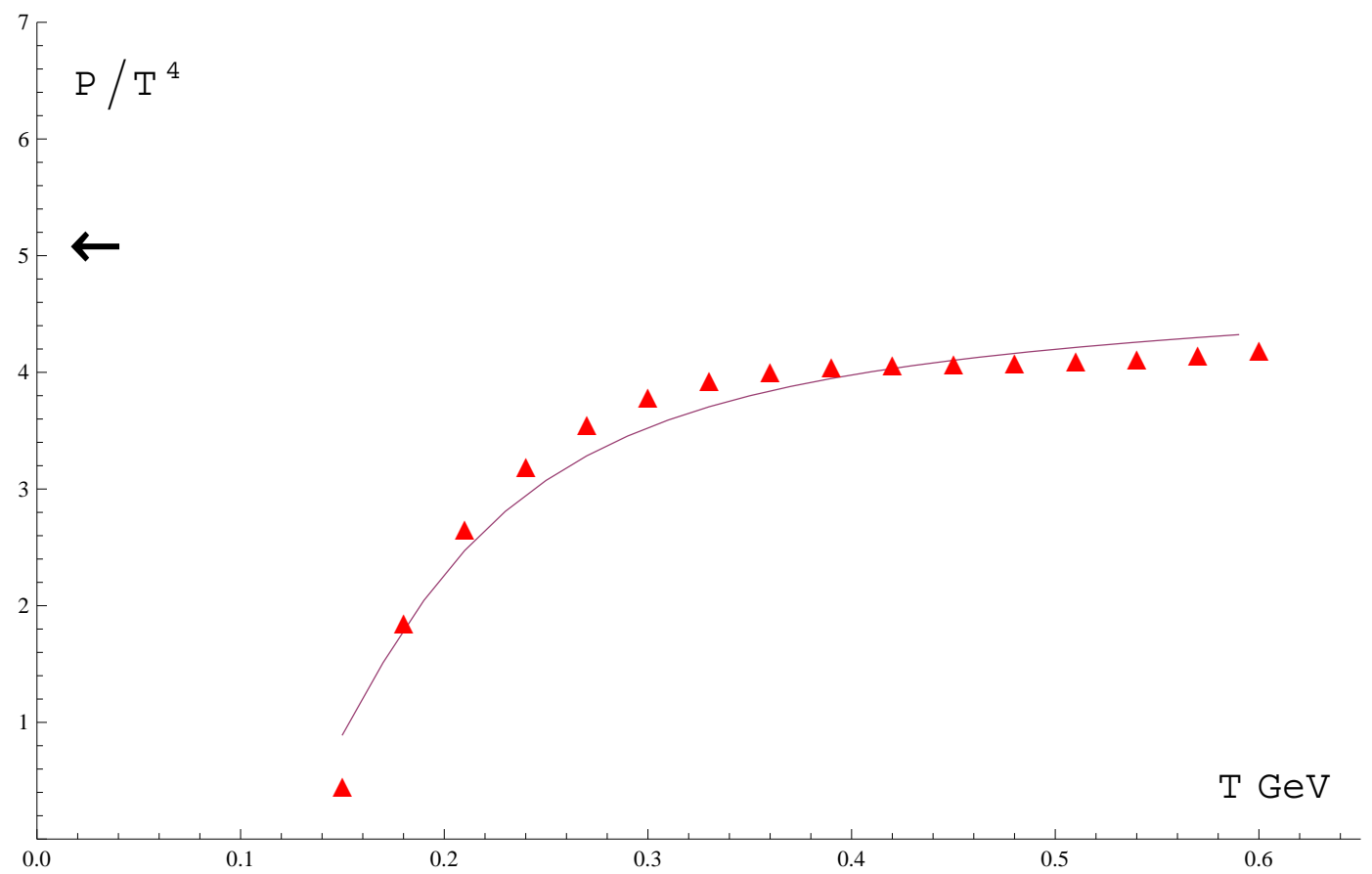

Figure 4. The solid curve gives the pressure normalized to $T^{4}$ comparing with lattice QCD results for two flavors $n_{f}=3$.

understood that the confining potential does not originate from quark-quark interaction, but it arises from the repulsion of colored quarks by the structured QCD vacuum state [22]. The positive value of bag pressure is coming from the difference in energy density between the QGP and the Hadronic states of matter. The negative value of bag pressure, in our opinion, may be understood by considering the simplest imagination of QGP formation, in which the bag surface is compressed towards the bag center and so it always reduce the diameter of the bag. Thus the negative sign is not a numerical value to the bag pressure, but it has a physical interpretation. Although we tried to fined a physical origin to the negative bag pressure, it still needs more study.

\subsection{Conclusion}

In this paper, the effects of Generalized Uncertainty Principle, which has been predicted by various theories of quantum gravity replacing the Heisenberg's uncertainty principle near the Planck scale, on the thermodynamics of ideal Quark-Gluon Plasma (QGP) consisting of three massless quark flavors is included. There is a clear effect on the thermodynamical quantities like the pressure and the energy density which means that a different effects from quantum gravity may be used in enhancement the theoretical results for Quark-Gluon Plasma state of matter. This effect looks like the techniqe used in lattice QCD. We determine the value of the bag parameter from fitting lattice QCD data and a physical interpretation to the negative bag pressure is introduced.

\section{Acknowledgements}

The research of NME, LIA, IE and AFA is supported by Benha University. IE would like to thank Prof. Abdel Nasser Tawfik for his fruitful discussion.

\section{REFERENCES}

[1] A. F. Ali, S. Das and E. C. Vagenas, "Discreteness of Space from the Generalized Uncertainty Principle," Physics Letters B, Vol. 678, No. 5, 2009, p. 497-499. doi:10.1016/i.physletb.2009.06.061

[2] A. F. Ali, S. Das and E. C. Vagenas, "The Generalized Uncertainty Principle and Quantum Gravity Phenomenology," 2010. arXiv:1001.2642[hep-th]

[3] S. Das, E. C. Vagenas and A. F. Ali, "Discreteness of Space from GUP II: Relativistic Wave Equations," Physics Letters B, Vol. 690, No. 4, 2010, p. 407-412. arXiv:1005.3368[hep-th]

[4] T. Thiemann, "A Length Operator for Canonical Quantum Gravity," Journal of Mathematical Physics, Vol. 39, No. 6, 1998, pp. 3372-3392. doi:10.1063/1.532445

[5] I. Pikovski, M. R. Vanner, M. Aspelmeyer, M. Kim and C. Brukner, "Probing Planck-Scale Physics with Quantum Optics," Nature Physics, Vol. 8, 2012, pp. 393-397. doi:10.1038/nphys2262

[6] S. Das and E. C. Vagenas, "Universality of Quantum Gravity Corrections," Physical Review Letters, Vol. 101, No. 22, 2008, Article ID: 221301. doi:10.1103/PhysRevLett.101.221301 
[7] S. Das and E. C. Vagenas, "Phenomenological Implications of the Generalized Uncertainty Principle," Canadian Journal of Physics, Vol. 87, No. 3, 2009, pp. 233-240. doi:10.1139/P08-105

[8] A. F. Ali, S. Das and E. C. Vagenas, "A Proposal for Testing Quantum Gravity in the Lab," Physical Review D, Vol. 84, 2011, Article ID: 044013. arXiv:1107.3164[hep-th]

[9] A. F. Ali, "Minimal Length in Quantum Gravity, Equivalence Principle and Holographic Entropy Bound," Classical and Quantum Gravity, Vol. 28, 2011, Article ID: 065013. arXiv:1101.4181[hep-th]

[10] R. Collela, A. W. Overhauser and S. A. Werner, "Observation of Gravitationally Induced Quantum Interference," Physical Review Letters, Vol. 34, No. 23, 1975, pp. 14721474. doi:10.1103/PhysRevLett.34.1472

[11] K. C. Littrell, B. E. Allman and S. A. Werner, "TwoWavelength-Difference Measurement of Gravitationally Induced Quantum Interference Phases," Physical Review A, Vol. 56, No. 3, 1997, pp. 1767-1780. doi:10.1103/PhysRevA.56.1767

[12] A. Camacho and A. Camacho-Galvan, "Test of Some Fundamental Principles in Physics via Quantum Interference with Neutrons and Photons," Reports on Progress in Physics, Vol. 70, 2007, pp. 1-56. arXiv:0810.1325[gr-qc]

[13] K. Yagi, T. Hatsuda and Y. Miake, "Quark-Gluon Plasma from Big Bang to Little Bang," Cambridge University Press, Cambridge, 2005.

[14] W.Greiner, L. Neise and H. Stocker, "Thermodynamics and Statistical Mechanics," 1997.
[15] J. Letessier and J. Rafelski, "Hadrons and Quark-Gluon Plasma," Cambridge University Press, Cambridge, 2004.

[16] A. S. Kapoyannis, "The Gibbs Equilibrium Conditions Applied to the QGP-Hadron Transition Curve," The European Physical Journal C, Vol. 51, No. 4, 2007, p. 1013. doi:10.1140/epjc/s10052-007-0374-8

[17] V. V. Begun, M. I. Gorenstein and O. A. Mogilevsky, "Modified Bag Models for the Quark Gluon Plasma Equation of State," International Journal of Modern Physics E, Vol. 20, 2011, pp. 1805-1815. arXiv:1004.0953v3[hep-ph]

[18] V. V. Begun, M. I. Gorenstein and O. A. Mogilevsky, "Equation of State for the Quark Gluon Plasma with the Negative Bag Constant," Ukrainian Journal of Physics, Vol. 55, No. 9, 2010, p. 1049. arXiv:1001.3139[hep-ph]

[19] M. I. Gorenstein and O. A. Mogilevsky, "On a Non-Perturbative Pressure Effect in Lattice QCD," Zeitschrift für Physik C Particles and Fields, Vol. 38, No. 1, 1988, pp. 161-163. doi:10.1007/BF01574531

[20] M. I. Gorenstein and S. N. Yang, "Gluon Plasma with a Medium-Dependent Dispersion Relation," Physical Review D, Vol. 52, No. 9, 1995, pp. 5206-5212. doi:10.1103/PhysRevD.52.5206

[21] F. Karsch, E. Laermann and A. Peikert, "The Pressure in 2, $2+1$ and 3 Flavour QCD," Physics Letters B, Vol. 478, No. 4, 2000, pp. 447-455. doi:10.1016/S0370-2693(00)00292-6

[22] P. N. Bogolioubov, "Sur un Modéle à Quarks QuasiIndépendants," Annales de l'Institut Henri Poincaré, Vol. 8, No. 2, 1967, pp. 163-189. 